\title{
Investigation of Homocysteine-Pathway-Related Variants in Essential Hypertension
}

\author{
Javed Y. Fowdar, Marta V. Lason, Attila L. Szvetko, Rodney A. Lea, and Lyn R. Griffiths \\ Genomics Research Centre, Griffith Health Institute, Gold Coast Campus, Griffith University, Southport, QLD 4222, Australia \\ Correspondence should be addressed to Lyn R. Griffiths, l.griffiths@griffith.edu.au
}

Received 21 March 2012; Revised 5 September 2012; Accepted 18 September 2012

Academic Editor: Thomas Unger

Copyright (๑) 2012 Javed Y. Fowdar et al. This is an open access article distributed under the Creative Commons Attribution License, which permits unrestricted use, distribution, and reproduction in any medium, provided the original work is properly cited.

\begin{abstract}
Hyperhomocysteinemia (hHcy) has been associated with an increased risk of cardiovascular disease and stroke. Essential hypertension (EH), a polygenic condition, has also been associated with increased risk of cardiovascular related disorders. To investigate the role of the homocysteine ( $\mathrm{Hcy}$ ) metabolism pathway in hypertension we conducted a case-control association study of Hcy pathway gene variants in a cohort of Caucasian hypertensives and age- and sex-matched normotensives. We genotyped two polymorphisms in the methylenetetrahydrofolate reductase gene (MTHFR C677T and MTHFR A1298C), one polymorphism in the methionine synthase reductase gene (MTRR A66G), and one polymorphism in the methylenetetrahydrofolate dehydrogenase 1 gene (MTHFD1 G1958A) and assessed their association with hypertension using chi-square analysis. We also performed a multifactor dimensionality reduction (MDR) analysis to investigate any potential epistatic interactions among the four polymorphisms and EH. None of the four polymorphisms was significantly associated with EH and although we found a moderate synergistic interaction between MTHFR A1298C and MTRR A66G, the association of the interaction model with EH was not statistically significant $(P=0.2367)$. Our findings therefore suggest no individual or interactive association between four prominent Hcy pathway markers and $\mathrm{EH}$.
\end{abstract}

\section{Introduction}

Hypertension is defined as a sustained systolic blood pressure of greater than $140 \mathrm{mmHg}$, a diastolic blood pressure of greater than $90 \mathrm{mmHg}$, or both [1]. Ninety five percent of hypertensives suffer from essential hypertension (EH) with the remaining $5 \%$ exhibiting high blood pressure due to some underlying disorder such as Liddle's syndrome, glucocorticoid-remediable aldosteronism, or apparent mineralocorticoid excess syndrome [1]. Worldwide, about one billion people suffer from hypertension while in Australia at least $30 \%$ of men and $20 \%$ of women are hypertensive [2]. In addition to the direct costs of treating EH, it is also a risk factor for many cardiovascular diseases (CVD), with $\mathrm{EH}$ implicated in 7.5 million deaths annually from ischaemic heart disease and stroke [3]. Determining the risk factors for $\mathrm{EH}$ is therefore important for understanding both $\mathrm{EH}$ and
CVD and may help to develop new treatment or prevention strategies.

There are a number of environmental and clinical risk factors associated with $\mathrm{EH}$ including, but not limited to, dietary intake of sodium, alcohol intake, lack of exercise, poor diet, obesity, insulin resistant diabetes, and hyperlipidemia. Although these factors explain a substantial proportion of hypertension susceptibility, it is estimated that up to $60 \%$ of the variation in hypertension risk is due to an individual's genetic makeup [4]. Thus, many studies have investigated the genetic component of hypertension using the well-known animal model, the spontaneous hypertensive rat [5], or undertaking genetic association and linkage studies [6] in hypertensive case-control and family cohorts. Investigations into the genetic component of hypertension have mainly focussed on the renin-angiotensin-aldosterone (RAA) system because of its importance in regulating normal 


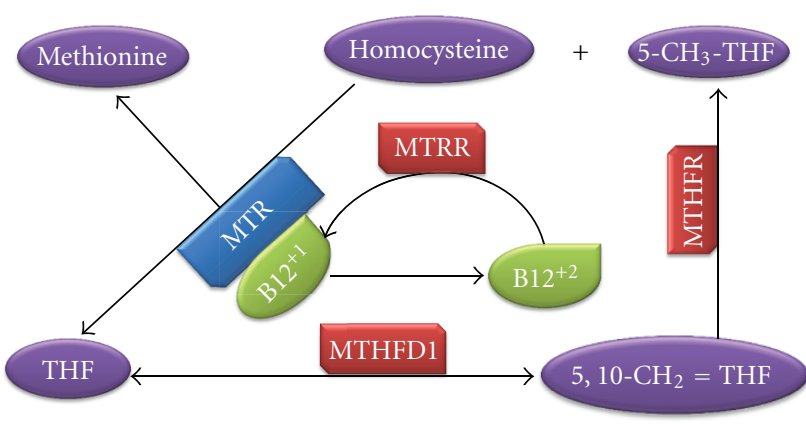

Figure 1: Simplified homocysteine pathway.

blood pressure [7]. Other genes, such as those involved in the central nervous system, vascular-endothelial system, and metabolic system, have also been extensively studied [7].

The homocysteine (Hcy) pathway has emerged as a strong candidate for $\mathrm{EH}$ and many studies have investigated genetic variation underlying hyperhomocysteinemia (hHcy). However, results have so far been inconclusive, with some studies reporting a significant association [8$10]$ while others have reported no association [11, 12]. The third National Health and Nutrition Examination Survey (NHANES III) reported that people with the highest level of Hcy carried a 2 to 3 fold increase in hypertension prevalence than those with the lowest Hcy level [13]. It is thought that Hcy levels are mainly increased by environmental factors such as lack of folate, vitamin B12, and vitamin $\mathrm{B} 6$ in the diet [14]; however, alterations in the Hcy pathway have also been shown to lead to mild hHcy in humans [15]. The Hcy pathway involves the conversion of Hcy to methionine. Briefly, tetrahydrofolate, a folic acid derivative, is converted to 5,10-methylenetetrahydrofolate (5,10-MTHF) by the enzyme methylenetetrahydrofolate dehydrogenase 1 (MTHFD1). 5,10-MTHF is converted to 5-methyltetrahydrofolate by methylenetetrahydrofolate reductase (MTHFR). Ultimately this substrate reacts with Hcy to form methionine and regenerates tetrahydrofolate. Methionine synthase (MTR), in the presence of cobalamin (vitamin B12), regulates this reaction. However over time, cobalamin which is a strong reductant becomes oxidised, thereby inactivating the MTR enzyme. The enzyme methionine synthase reductase (MTRR) reactivates MTR by reducing cobalamin to its original state [16]. A simplified pathway is shown in Figure 1.

One of the most studied genetic variants contributing to $\mathrm{hHcy}$ is the $\mathrm{C}$ to $\mathrm{T}$ single nucleotide polymorphism (SNP) at codon 677 of the MTHFR gene. The $\mathrm{C}$ to T substitution causes alanine to be substituted by valine. The TT variant codes for a thermolabile enzyme which has a $50 \%$ reduced activity compared to the CC variant [17]. Another SNP in the same gene occurs at codon 1298 with an A to C substitution. This leads to glutamine being substituted by alanine. Although the $\mathrm{CC}$ variant also reduces enzymatic activity, with its effect not as drastic as the TT variant occurring at codon 677 [18], both polymorphisms result in a decrease in MTHFR enzyme activity, which decreases production of 5-methyltetrahydrofolate, the necessary substrate for Hcy conversion to methionine. By decreasing levels of 5-methyltetrahydrofolate, these polymorphisms could therefore result in accumulation of Hcy, leading to hHcy [15].

Although the MTRR enzyme does not directly participate in the conversion of Hcy to methionine, the fact that it keeps the MTR enzyme active makes it a key enzyme in Hcy metabolism. A common SNP in MTRR is the A to $G$ substitution at codon 66. This substitution causes isoleucine to be substituted by methionine in the enzyme. It has been reported that the mutant enzyme exhibits a four-fold lower activity in reactivating MTR than the wild type enzyme [19]. This polymorphism has also been associated with increased Hcy levels [20]. The MTHFD1 gene codes for a tri-functional enzyme: 5,10MTHF dehydrogenase, 5,10-MTHF cyclohydrolase, and 10formyltetrahydrofolate synthetase. The G1958A polymorphism results in the replacement of arginine by glycine within the synthetase active domain and reduces the enzymatic activity of MTHFD1 by about $26 \%$ [16], thereby disrupting methionine synthesis and possibly resulting in increased levels of Hcy.

This study investigated whether there is an association between EH and the MTHFR C677T, MTHFR A1298C, MTRR A66G, and MTHFD1 G1958A variants in an Australian case-control cohort. An interaction analysis using the multifactor dimensionality reduction (MDR) method was also performed to investigate whether specific combinations of genotypes across all four loci contribute to disease status.

MDR analysis is a data mining method used to detect and classify combinations of independent variables such as genotypes or environmental factors that may interact to cause disease. MDR classifies the genotype combinations of two loci (multilocus genotype) into either belonging to a low-risk group or a high-risk group. For example, all possible genotypes at locus 1 (AA, Aa, aa) are paired with each other possible genotype at locus $2(\mathrm{BB}, \mathrm{Bb}, \mathrm{bb})$, giving nine possible multilocus genotypes $(\mathrm{AA} / \mathrm{BB}, \mathrm{AA} / \mathrm{Bb}, \mathrm{AA} / \mathrm{bb}$, and so on). Each multilocus genotype is then evaluated for the number of cases versus controls, and assigned to be highrisk if the number of cases exceeds the number of controls, corresponding to a ratio $>1$ for matched populations [21, 22 ]. If the ratio is $<1$, the multilocus genotype is defined as low-risk. When numbers are equal, multilocus genotypes can be assigned as affected (high risk), unaffected (low risk), or unassigned. This redefinition of two-dimensional (twolocus) data as one dimension (risk value) is how MDR reduces the complexity of multidimensional data. The risk value dimension can then be analysed to predict the outcome variable (case or control status) using a non-parametric method which is better suited to deal with modelling of high-order interactions in small sample sizes. Nonparametric methods such as MDR are being increasingly used for genetic interaction analysis as they are model-free and are considered more robust than parametric methods [22]. 


\section{Methods}

2.1. Study Population. The study protocol was approved by the Griffith University's Ethics Committee. The study population was composed of 409 hypertensives and 409 age- ( \pm 5 years $)$, sex-, and ethnicity-matched normotensive controls, who resided in the South East Queensland region of Australia. All participants were of Caucasian origin. Cases were defined as individuals who were clinically diagnosed as suffering from hypertension and who were taking antihypertensive drugs. Controls were defined as participants who were not taking antihypertensive drugs, and whose blood pressure was less than $140 / 90 \mathrm{mmHg}$. Individuals suffering from renal disorders (polycystic kidneys, renovascular disease, parenchymal renal disease), primary aldosteronism, Cushing syndrome, and hypothyroidism were excluded from the study. None of the participants included in the study reported any previous cardiovascular events such as heart attacks or stroke. $53.3 \%$ of the population were female and $46.7 \%$ were male. The average age of the case group was $63.1 \pm 10.9$ years and the average age of the control group was $61.0 \pm 10.5$ years. Peripheral blood samples as well as questionnaires detailing medical history, including blood pressure and prescribed medications, were obtained from all participants. All participants signed informed consent agreements prior to collection of blood and clinical information.

2.2. Genotyping Methods. DNA was extracted from blood samples using a modified version of the salting-out method [23]. Two polymorphisms in MTHFR and one polymorphism in MTRR and MTHFD1 were genotyped for all cases and controls. Detailed information regarding polymorphisms and a summary of assay conditions and primer sequences for each polymorphism are listed in Table 1. All PCR buffers, $\mathrm{MgCl}_{2}$, GoTaq polymerase were from Promega Corp., Madison, WI, USA; dNTPs, restriction enzymes, and enzyme buffers were from New England Biolabs, Ipswich, MA, USA; SYTO9 dye was from Invitrogen, Carlsbad, CA, USA. Protocol and assays for each polymorphism are described in detail below.

2.3. MTHFR Genotyping. The MTHFR C677T polymorphism was genotyped by polymerase chain reaction (PCR) followed by restriction fragment length polymorphism (RFLP) analysis. The PCR protocol was as follows: 1X PCR buffer, $1.75 \mathrm{mM} \mathrm{MgCl}_{2}, 0.2 \mathrm{mM}$ dNTPs, $0.2 \mathrm{uM}$ forward primer, $0.2 \mathrm{uM}$ reverse primer, $1 \mathrm{U}$ GoTaq, and $40 \mathrm{ng}$ of DNA. The primer sequences were designed by Frosst [24] and were validated as described in a previous study [25]. The PCR thermocycling conditions were as follows: $95^{\circ} \mathrm{C}$ for $3 \mathrm{mins}$, then $94^{\circ} \mathrm{C}$ for 40 seconds, $69^{\circ} \mathrm{C}$ for 40 seconds, and $72^{\circ} \mathrm{C}$ for 1 minute for 35 cycles, followed by a final extension step of $72^{\circ} \mathrm{C}$ for 5 minutes. The $198 \mathrm{bp}$ PCR products were electrophoresed on a $15 \mathrm{~cm} 2 \%$ agarose gel containing $0.006 \%$ ethidium bromide) for $30 \mathrm{mins}$ at $90 \mathrm{~V}$, and then visualised under ultraviolet light. $10 \mathrm{uL}$ of PCR product was then digested with $4 \mathrm{U} \operatorname{Hinf} \mathrm{I}$ and $1 \mathrm{X}$ NEB Buffer 2 at $37^{\circ} \mathrm{C}$ for $12 \mathrm{hrs}$, followed by an $80^{\circ} \mathrm{C}$ enzyme deactivation step of 20 mins. Restriction digest products were electrophoresed on a $15 \mathrm{~cm} 3.5 \%$ agarose gel for $120 \mathrm{~min}$ at $80 \mathrm{~V}$, which was then poststained in a $0.01 \%$ solution of ethidium bromide in 1X TAE buffer for $40 \mathrm{~min}$ and visualised under ultraviolet light. Hinf I digestion of fragments containing the $\mathrm{T}$ allele produced two fragments of $175 \mathrm{bp}$ and $23 \mathrm{bp}$ while fragments containing the $\mathrm{C}$ allele remained undigested by Hinf I.

The MTHFR A1298C polymorphism was genotyped by PCR followed by high resolution melt (HRM) analysis. The PCR protocol was as follows: 1X PCR buffer, $1.5 \mathrm{mM}$ $\mathrm{MgCl}_{2}, 0.2 \mathrm{mM}$ dNTPs, $0.3 \mathrm{uM}$ forward primer, $0.3 \mathrm{uM}$ reverse primer, 1.6uM SYTO9, 1 U GoTaq, and 40 ng of DNA. The primer sequences were obtained from a previous study [26] and were validated using an RFLP approach to genotype positive controls as described previously [27]. The PCR followed by high resolution melting analysis was conducted on a Qiagen Rotor-Q (Qiagen, Doncaster, VIC, Australia) and the thermocycling conditions were as follows: $95^{\circ} \mathrm{C}$ for 5 mins, then $95^{\circ} \mathrm{C}$ for 5 seconds and $60^{\circ} \mathrm{C}$ for 10 seconds for 45 cycles. PCR products were melted from $78^{\circ} \mathrm{C}$ to $88^{\circ} \mathrm{C}$ at $0.1^{\circ} \mathrm{C}$ increments every 2 seconds. Amplicon melting temperature (Tm) occurred at $83^{\circ} \mathrm{C}$ and three separate melt curves were obtained corresponding to the three genotypes $\mathrm{AA}, \mathrm{AC}$, and CC.

2.4. MTRR Genotyping. The MTRR A66G polymorphism was genotyped by PCR followed by HRM analysis. The PCR protocol was as follows: 1X PCR buffer, $1.5 \mathrm{mM} \mathrm{MgCl}_{2}$, $0.2 \mathrm{mM}$ dNTPs, $0.3 \mathrm{uM}$ forward primer, $0.3 \mathrm{uM}$ reverse primer, 1.6 uM SYTO9, 1U GoTaq. The primer sequences were obtained from a previous study [28] and were validated using an RFLP approach described previously [27]. The PCR followed by HRM analysis was conducted on a Qiagen Rotor-Q and the thermocycling conditions were as follows: $95^{\circ} \mathrm{C}$ for 5 mins, then $95^{\circ} \mathrm{C}$ for 5 seconds and $60^{\circ} \mathrm{C}$ for 10 seconds for 45 cycles. PCR products were melted from $75^{\circ} \mathrm{C}$ to $85^{\circ} \mathrm{C}$ at $0.1^{\circ} \mathrm{C}$ increments every 2 seconds. Amplicon Tm occurred at $80^{\circ} \mathrm{C}$ and three separate melt curves were obtained corresponding to the three genotypes AA, AG, and GG.

2.5. MTHFD1 Genotyping. The MTHFD1 G1958A polymorphism was genotyped by PCR followed by HRM analysis. The PCR protocol was as follows: 1X PCR buffer, $1.5 \mathrm{mM}$ $\mathrm{MgCl}_{2}, 0.2 \mathrm{mM}$ dNTPs, $0.3 \mathrm{uM}$ forward primer, $0.3 \mathrm{uM}$ reverse primer, $1.6 \mathrm{uM}$ SYTO9, $1 \mathrm{U}$ GoTaq. The primer sequences were obtained from a previous study [29] and were validated using an RFLP approach to genotype positive controls as described previously [29]. The PCR followed by HRM analysis was conducted on a Qiagen Rotor-Q and the thermocycling conditions were as follows: $95^{\circ} \mathrm{C}$ for $5 \mathrm{mins}$, then $95^{\circ} \mathrm{C}$ for 5 seconds and $60^{\circ} \mathrm{C}$ for 10 seconds for 45 cycles. PCR products were melted from $79^{\circ} \mathrm{C}$ to $89^{\circ} \mathrm{C}$ at $0.1^{\circ} \mathrm{C}$ increments every 2 seconds. Amplicon Tm occurred at $84^{\circ} \mathrm{C}$ and three separate melt curves were obtained corresponding to the three genotypes AA, AG, and GG. 
2.6. Statistical Analysis. Power analysis for this study was performed using the Power for Genetic Analyses software [30]. Genotype counts were tabulated for each of the four markers and genotype and allele frequencies were computed for each marker. All groups were tested for and found to be within Hardy-Weinberg equilibrium (HWE). Genotype and allele frequencies were compared between case and control groups for each marker using the chi-square test, with two and one degrees of freedom, respectively. All statistical analyses were performed using Microsoft Excel 2010 for Windows (v14.0).

2.7. Interaction Analysis. Given the possibility that each variant may only contribute a small independent effect which may not be detectable as statistically significant in our case control cohort, we also performed interaction analysis using the MDR 2.0 software version beta 8.4. The MDR program was designed to test for interactive genetic effects on a trait even if the independent effects are nonsignificant [22].

In the MDR software, main effect (one-locus) models, two-locus models, or N-locus models are generated, and each model is assessed for prediction accuracy by dividing the dataset into multiple sets, with one set excluded from model-training and then used to test the model. The process of division, model-training, and model-testing is repeated multiple times to cross-validate each model. Testing accuracy (TA) and cross-validation consistency (CVC) are then used to evaluate the overall best model. Permutation testing can then be performed on the dataset using an additional module called MDRpt, which evaluates the significance of the model TA [22].

Before performing the MDR analysis, all markers were examined for correlation using PLINK's pairwise LD function [31], to identify SNPs that may be collinear. None of the four markers were found to be significantly correlated $\left(r^{2}>0.85\right)$ and all were used in the MDR analysis. Missing genotypes were then imputed by mode substitution. Software default settings were used except that the crossvalidation was repeated 100 times, and paired analysis was selected. The model with the highest TA and CVC was determined to be the best model and significance $P$ values were then generated using 10,000 permutations in the MDR permutation testing module (MDRpt) version 1.0 beta 2 .

\section{Results}

This study has more than $90 \%$ power to detect a relative risk of at least 1.5 for all markers. Genotype and allele frequencies for all four markers are shown in Table 2. Of the 409 cases and 409 controls, 377 cases (92.2\%) and 393 controls $(96.1 \%)$ and 368 cases $(90.0 \%)$ and 386 controls (94.4\%) were successfully genotyped for the MTHFR C677T and MTHFR A1298C markers, respectively. For the MTRR A66G marker, 360 cases $(88.0 \%)$ and 358 controls $(87.5 \%)$ were successfully genotyped, and for the MTHFD1 marker, 364 cases $(89.0 \%)$ and 360 controls $(88.0 \%)$ were successfully genotyped. Samples which exhibited ambiguous melt curves for high resolution melt analysis were not counted resulting in a lower genotyping success rate compared to the RLFP assay. Both case and control groups across all four markers were found to be in $\operatorname{HWE}(P>0.05)$.

For MTHFR, there was no statistically significant difference between the genotype frequencies of cases and controls for either the C677T marker $\left(\chi^{2}=0.03, P=0.99\right)$ or the A1298C marker $\left(\chi^{2}=1.10, P=0.58\right)$. There was also no statistically significant difference between the allele frequencies of cases and controls for either the C677T $\left(\chi^{2}=0.02, P=\right.$ $0.88)$ or the A1298C $\left(\chi^{2}=0.16, P=0.69\right)$ polymorphisms. For the C677T marker, there was no observed trend in either the genotype or allele frequencies, with the TT genotype frequency at $8.7 \%$ for cases and $8.9 \%$ for controls, and the $\mathrm{T}$ allele frequency at $31.8 \%$ for cases and $32.2 \%$ for controls. For the A1298C marker, there was an increased AA genotype frequency in cases $(44.8 \%)$ compared to controls $(42.0 \%)$, though this trend was less apparent in A allele frequency in cases $(65.4 \%)$ compared to controls $(64.4 \%)$. The observed minor allele frequencies in the control group for both the C677T marker ( $\mathrm{T}$ allele, 32.2\%) and the A1298C marker (C allele, 35.6\%) conformed well with expected control frequencies for each marker (C677T, T allele, 31\%; A1298C, C allele, 36\%) as determined in the Hap-Map CEU population (Utah residents of Northern European ancestry).

Similarly, for the MTRR A66G polymorphism, there was no statistically significant difference between either the genotype frequencies of cases and controls $\left(\chi^{2}=0.92, P=\right.$ $0.63)$, or the allelic frequencies of cases and controls $\left(\chi^{2}=\right.$ $0.79, P=0.37)$. The GG genotype frequency was $18.1 \%$ for cases and $20.7 \%$ for controls, while allele frequencies showed a trend of decreased $G$ allele frequency in cases $(44.7 \%)$ compared to controls $(47.1 \%)$. Although the genotype frequencies of our control group seemed markedly different to the Hap-Map CEU frequencies with $52.8 \%$ of heterozygotes in our control population compared to only $34.0 \%$ in the Hap-Map CEU population, the allelic frequencies of our control group (A allele, 52.9\%) and the Hap-Map CEU population (A allele, 55.0\%) were similar.

For the MTHFD1 G1958A polymorphism, there was no statistically significant difference between cases and controls for either the genotype frequencies $\left(\chi^{2}=1.73, P=0.42\right)$ or the allelic frequencies of cases and controls $\left(\chi^{2}=0.31\right.$, $P=0.58)$. The GG genotype frequency was $32.7 \%$ for cases and $28.9 \%$ for controls, while the G allele frequency was $55.8 \%$ for cases and $54.3 \%$ for controls. The observed allele frequencies for our control group ( $\mathrm{G}$ allele, 54.3\%) was similar to expected allele frequencies as determined by the Hap-Map CEU population (G allele, 58.0\%). Case and control genotype frequencies were also analysed by gender (Table 3 ). There were 436 females (218 cases and controls) and 382 males (191 cases and controls); all groups were found to be in HWE. No significant differences between cases and controls were detected when analysed by gender and therefore all further analyses were performed using the entire population.

For the MDR analysis, the best MDR models for the one SNP (main effect), two SNP, and three SNP combinations are shown in Table 4 . The best model had a TA of 0.5526 and CVC of 100/100, and was a two-SNP model containing 

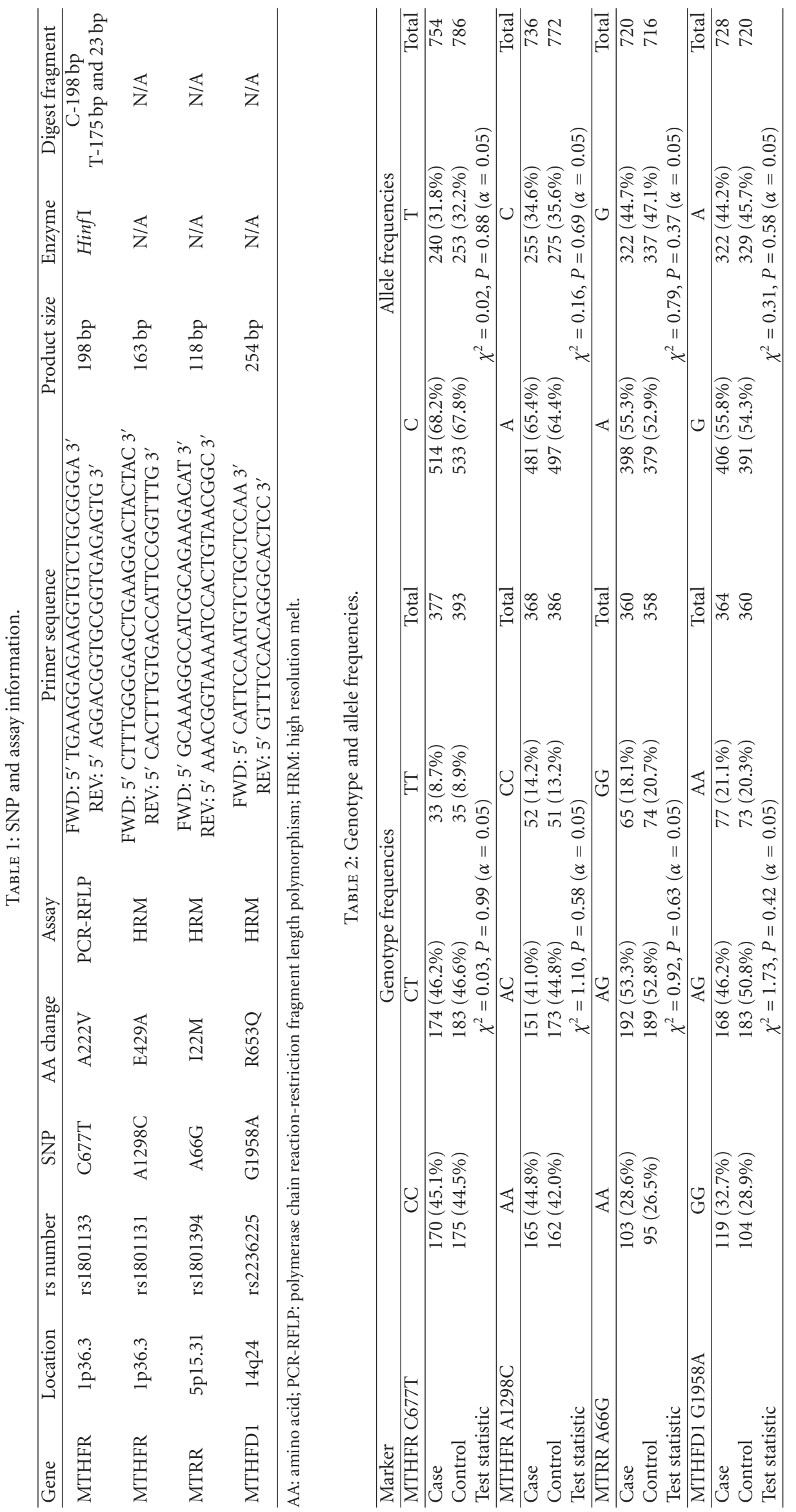


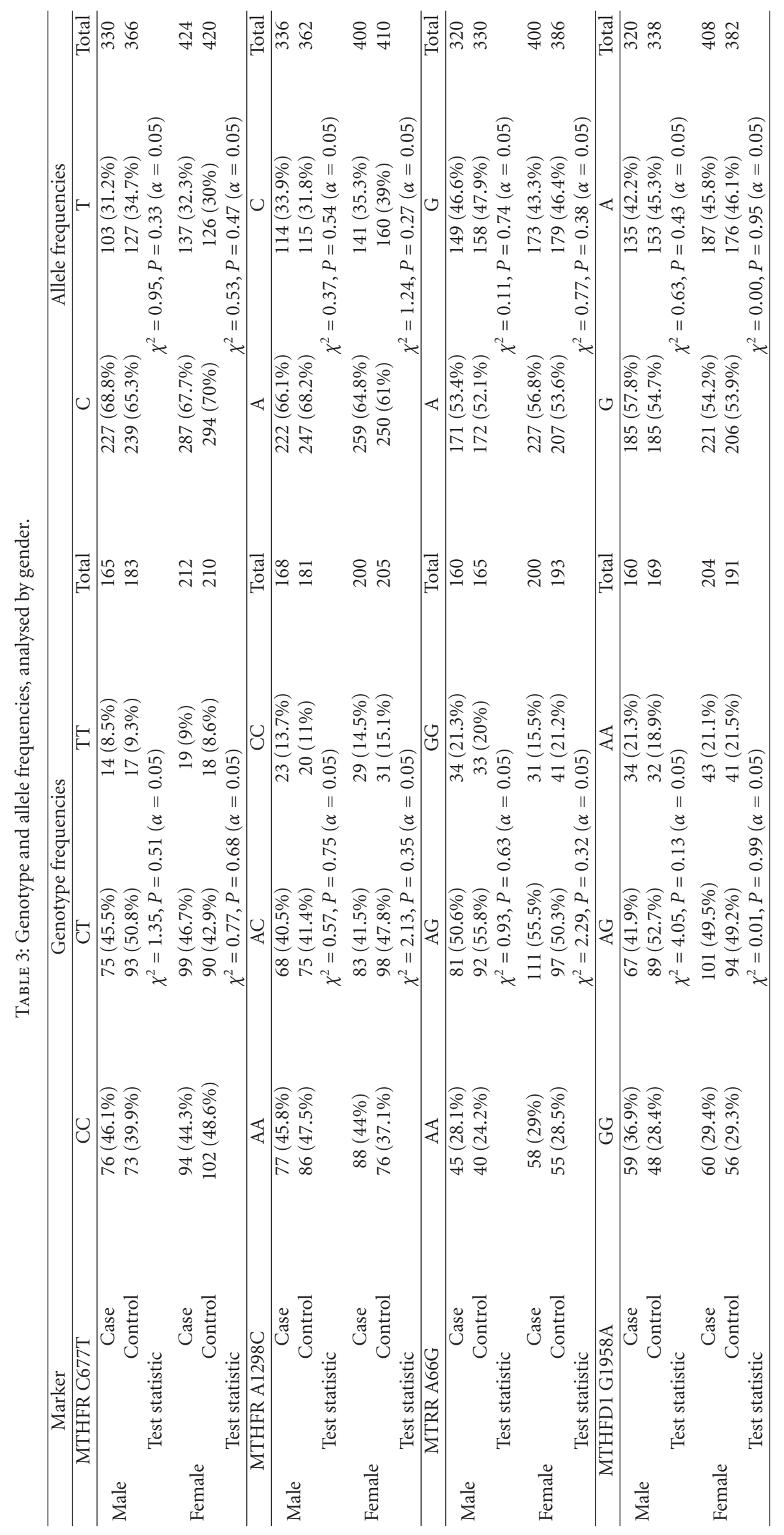


TABle 4: Best MDR models.

\begin{tabular}{|c|c|c|c|c|}
\hline Model & Training accuracy & Testing accuracy & CV consistency & $P$ value \\
\hline MTHFR1298 & 0.5270 & 0.4951 & $97 / 100$ & 0.9621 \\
\hline MTHFR1298_MTRR & 0.5575 & 0.5526 & $100 / 100$ & 0.2367 \\
\hline MTHFR677_MTHFR1298_MTRR & 0.5681 & 0.4780 & $68 / 100$ & 0.9863 \\
\hline
\end{tabular}

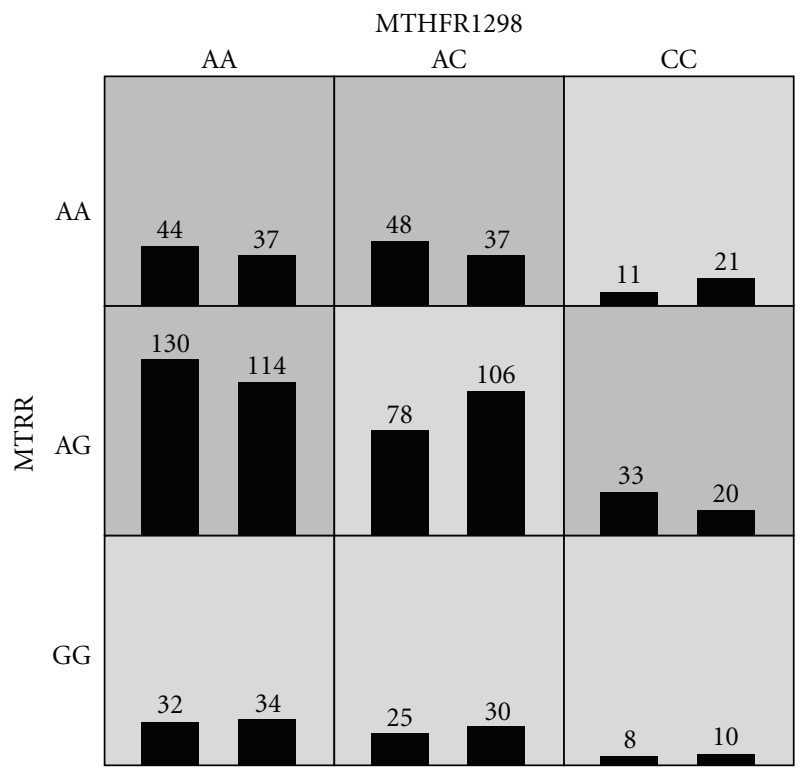

FIgURE 2: Frequencies of cases and controls for the best MDR model (MTHFR1298-MTRR). Low-risk combined genotypes are indicated by light grey cells and high-risk combined genotypes are indicated by dark grey cells.

the MTHFR1298 and MTRR markers. Figure 2 shows the frequency of cases and controls for each multilocus genotype in the model. The light grey cells indicate genotype combinations (MTHFR1298-MTRR) of the low risk group and the dark grey cells indicate genotype combinations of the high risk group. When multidimensional data under the MTHFR1298-MTRR model were collapsed into one dimension (risk level), the frequency of controls was higher in the low-risk group compared to cases (201 controls, 154 cases) while case frequency was higher in the highrisk group compared to controls (255 cases, 208 controls). There appears to be a moderate synergistic effect between MTHFR1298 and MTRR and a weaker synergistic effect between MTHFR677 and MTHFD1. However, the best model (MTHFR1298-MTRR model) was found not to be significantly associated with case status $(P=0.2367)$.

\section{Discussion}

We investigated the homocysteine pathway variants MTHFR C677T, MTHFR A1298C, MTRR A66G, and MTHFD1
G1598A in an Australian Caucasian population for association with $\mathrm{EH}$. There was no statistical difference between our case and control groups for either genotype or allele frequencies for any of the markers studied, indicating no detected association between these four markers and $\mathrm{EH}$ in our case-control population. However, given the sample size limitation, we could not rule out the possibility that these variants contributed a modest effect on $\mathrm{EH}$ in this cohort $(\mathrm{OR}<1.5)$ that was not detectable as statistically significant in this study, therefore, we conducted the interaction analysis using an MDR approach. We found that the best model indicated an interaction between the two SNPs MTHFR A1298C and MTRR A66G, which was found to be nonsignificant by permutation testing. This may reflect the fact that the mechanism by which hHcy can cause hypertension is not well understood. However, a recent study in human umbilical artery smooth muscle cells reported an increase in the proliferation of vascular smooth muscle cells through the Hcy-mediated differential regulation of cyclin A and D1, which led to an increase in intima media thickness [32]. Another study on mesenteric arteries in mice showed that hHcy decreased bioavailability of nitric oxide by decreasing the expression of endothelial nitric oxide synthase through the activation of matrix metalloproteinases during oxidative stress [33]. These studies seem to implicate hHcy in vascular remodelling or vasoconstriction, suggesting a possible mechanism for $\mathrm{EH}$ development.

MTHFR has been among the most studied genes in relation to Hcy and folate metabolism, with regard to a variety of diseases ranging from neural tube defects to CVD and EH. Previous studies have shown that the MTHFR variants C677T and A1298C have been associated with both higher levels of Hcy [15] and EH risk [34] directly. Currently, MTHFR C677T has been studied in relation to hypertension in 29 published papers indexed on the PubMed database, 25 of which were included in a meta-analysis conducted in 2007, which concluded that there was an overall association of MTHFR C677T with hypertension, with an OR of 1.343 (95\%CI 1.198-1.505) [35]. Overall, this is less than a two fold increase in OR for EH cases, which may indicate that larger sample sizes would be needed to detect a modest effect. However, the sample size for this study (409 cases, 409 controls) is larger than the largest study included in the meta-analysis (247 cases, 249 controls). The metaanalysis also showed high heterogeneity between studies, with only 6 published studies showing a clear statistically significant association with $\mathrm{EH}$, while 19 published studies had a nonsignificant OR [35]. However, studies included were from various countries and ethnicities, suggesting that 
population differences in allele frequency and association may have been confounded.

Another meta-analysis of Hcy metabolizing enzymes and risk of coronary heart disease consisting of 23 studies reported an association of the C allele of the MTHFR A1298C with myocardial infarction with an OR of 1.37 (95\% CI 1.03-1.84) [36]. However, conflicting results were obtained when the controls were subdivided and analysed with the $\mathrm{C}$ allele being associated with a decreased risk of coronary heart disease (CHD) in hospital-based case-control studies while it was associated with an increased risk of CHD in population-based case-control studies [36]. Overall, findings for MTHFR have therefore been varied and may represent differing MTHFR allele frequencies between ethnic groups, low power of small studies to detect modest effect sizes on CVD and EH risk, or a true lack of association between MTHFR variants and CVD and EH.

MTRR and MTHFD1 have both been shown to carry variants which decrease enzymatic activity and disrupt either MTR reactivation (for MTRR) or purine synthesis (for MTHFD1) though MTHFD1 has not been previously studied in relation to EH. The MTRR A66G polymorphism has been associated with increased Hcy levels [20]. However, a recent study of the MTRR A66G marker reported a lack of association with both Hcy concentration and risk of vascular disease [37], and a 2002 study in adolescents failed to find an association with $\mathrm{EH}$ [38]. This is the first study which has examined both MTRR and MTHFD1 in association with adult $\mathrm{EH}$, and though individually they do not appear significantly associated with $\mathrm{EH}$ risk, it is possible that each variant confers only a modest effect. We hypothesised that an interaction analysis may have greater power to detect tiny effect sizes for each marker, and therefore conducted an interaction analysis using MDR. Though synergistic effects were detected, especially between MTHFR A1298C and MTRR, the best model was not found to be significant and therefore these effects may not be due to a true interaction between the variants, or may need to be confirmed in a larger case-control cohort. The interaction analysis did not detect MTHFR C677T as part of the best model, which is unexpected as the strongest individual association has been previously found between this variant and EH [35]; however, this may be because MTHFR C677T is not significantly associated with $\mathrm{EH}$ in this population.

Current data from this and other studies suggest that genes within the Hcy pathway are not significantly associated with an increase in EH risk, including the well-studied marker MTHFR C677T. Additionally, given that each gene may confer a modest effect to $\mathrm{EH}$ risk, a polygenic profile analysis of genes in the Hcy pathway may be warranted. Additionally, future studies should measure plasma Hcy levels to determine whether a combination of these markers influences Hcy levels overall. Our study could not verify whether Hcy levels are significantly different between our cases and controls, and whether individual markers or combinations of markers influence EH risk through elevating Hcy levels. Further, the effects of diet on Hcy levels and $\mathrm{EH}$ risk should be controlled for in any future analysis as protective diet such as high folate intake may abrogate an increased genetic risk to $\mathrm{EH}$ due to genetic variations in the Hcy pathway.

\section{Conflict of Interests}

The authors declare no conflict of interests.

\section{Acknowledgments}

The authors would like to thank Ms. Rebecca Grealy for her comments on the paper. J. Fowdar has been funded by an Endeavour International Postgraduate Research Scholarship (EIPRS) and a Griffith University Postgraduate Research Scholarship (GUPRS). This research received no specific grant from any funding agency in the public, commercial, or not-for-profit sectors.

\section{References}

[1] A. V. Chobanian, G. L. Bakris, H. R. Black et al., "The 7th report of the joint national committee on prevention, detection, evaluation, and treatment of high blood pressure: the JNC 7 report," Journal of the American Medical Association, vol. 289, no. 19, pp. 2560-2572, 2003.

[2] P. M. Kearney, M. Whelton, K. Reynolds, P. Muntner, P. K. Whelton, and J. He, "Global burden of hypertension: analysis of worldwide data," Lancet, vol. 365, no. 9455, pp. 217-223, 2005.

[3] G. A. Mensah and G. Bakris, "The United Nations high level meeting addresses noncommunicable diseases, but where is hypertension?" The Journal of Clinical Hypertension, vol. 13, no. 11, pp. 787-790, 2011.

[4] M. Singh, G. A. Mensah, and G. Bakris, "Pathogenesis and clinical physiology of hypertension," Cardiology Clinics, vol. 28 , no. 4, pp. 545-559, 2010.

[5] M. Pravenec, "Use of rat genomics for investigating the metabolic syndrome," Methods in Molecular Biology, vol. 597, pp. 415-426, 2010.

[6] C. Delles, M. W. McBride, D. Graham, S. Padmanabhan, and A. F. Dominiczak, "Genetics of hypertension: from experimental animals to humans," Biochimica et Biophysica Acta, vol. 1802, no. 12, pp. 1299-1308, 2010.

[7] S. J. Lupton, C. L. Chiu, and J. M. Lind, "A hypertension gene: are we there yet?" Twin Research and Human Genetics, vol. 14, no. 4, pp. 295-304, 2011.

[8] G. Vyssoulis, E. Karpanou, S. M. Kyvelou et al., "Associations between plasma homocysteine levels, aortic stiffness and wave reflection in patients with arterial hypertension, isolated office hypertension and normotensive controls," Journal of Human Hypertension, vol. 24, no. 3, pp. 183-189, 2010.

[9] V. A. M. Garfunkel, P. I. Porto, S. I. Garcia et al., "Hyperhomocysteinemia but not MTHFR genotype is associated with young-onset essential hypertension," Journal of Human Hypertension, vol. 17, no. 5, pp. 361-364, 2003.

[10] G. Y. Lip, E. Edmunds, S. C. Martin, A. F. Jones, A. D. Blann, and D. G. Beevers, "A pilot study of homocyst(e)ine levels in essential hypertension: relationship to von Willebrand factor, an index of endothelial damage," American Journal of Hypertension, vol. 14, no. 7, pp. 627-631, 2001.

[11] T. S. Bowman, J. M. Gaziano, M. J. Stampfer, and H. D. Sesso, "Homocysteine and risk of developing hypertension in men," 
Journal of Human Hypertension, vol. 20, no. 8, pp. 631-634, 2006.

[12] C. van Guldener, P. W. Nanayakkara, and C. D. Stehouwer, "Homocysteine and blood pressure," Current Hypertension Reports, vol. 5, no. 1, pp. 26-31, 2003.

[13] U. Lim and P. A. Cassano, "Homocysteine and blood pressure in the 3rd National Health and Nutrition Examination Survey, 1988-1994," American Journal of Epidemiology, vol. 156, no. 12, pp. 1105-1113, 2002.

[14] A. Virdis, L. Ghiadoni, G. Salvetti, D. Versari, S. Taddei, and A. Salvett, "Hyperhomocyst(e)inemia: is this a novel risk factor in hypertension?" Journal of Nephrology, vol. 15, no. 4, pp. 414421, 2002.

[15] A. K. Böttiger, A. Hurtig-Wennlöf, M. Sjöström, A. Yngve, and T. K. Nilsson, "Association of total plasma homocysteine with methylenetetrahydrofolate reductase genotypes $677 \mathrm{C}>\mathrm{T}$, $1298 \mathrm{~A}>\mathrm{C}$, and $1793 \mathrm{G}>\mathrm{A}$ and the corresponding haplotypes in Swedish children and adolescents," International Journal of Molecular Medicine, vol. 19, no. 4, pp. 659-665, 2007.

[16] K. E. Christensen, C. V. Rohlicek, G. U. Andelfinger et al., "The MTHFD1 p.Arg653Gln variant alters enzyme function and increases risk for congenital heart defects," Human Mutation, vol. 30, no. 2, pp. 212-220, 2009.

[17] C. Konrad, G. A. Müller, C. Langer et al., "Plasma homocysteine, MTHFR C677T, CBS 844ins68bp, and MTHFD1 G1958A polymorphisms in spontaneous cervical artery dissections," Journal of Neurology, vol. 251, no. 10, pp. 1242-1248, 2004.

[18] R. Palomino-Morales, C. Gonzalez-Juanatey, T. R. VazquezRodriguez et al., "A1298C polymorphism in the MTHFR gene predisposes to cardiovascular risk in rheumatoid arthritis," Arthritis Research and Therapy, vol. 12, no. 2, article 71, 2010.

[19] H. Olteanu, T. Munson, and R. Banerjee, "Differences in the efficiency of reductive activation of methionine synthase and exogenous electron acceptors between the common polymorphic variants of human methionine synthase reductase," Biochemistry, vol. 41, no. 45, pp. 13378-13385, 2002.

[20] D.J. Gaughan, "The methionine synthase reductase (MTRR) A66 G polymorphism is a novel genetic determinant of plasma homocysteine concentrations," Atherosclerosis, vol. 157, no. 2, pp. 451-456, 2001.

[21] A. G. Heidema, E. J. Feskens, P. A. Doevendans et al., "Analysis of multiple SNPs in genetic association studies: comparison of three multi-locus methods to prioritize and select SNPs," Genetic Epidemiology, vol. 31, no. 8, pp. 910-921, 2007.

[22] J. H. Moore, J. C. Gilbert, C. T. Tsai et al., "A flexible computational framework for detecting, characterizing, and interpreting statistical patterns of epistasis in genetic studies of human disease susceptibility," Journal of Theoretical Biology, vol. 241, no. 2, pp. 252-261, 2006.

[23] S. A. Miller, D. D. Dykes, and H. F. Polesky, "A simple salting out procedure for extracting DNA from human nucleated cells," Nucleic Acids Research, vol. 16, no. 3, article 1215, 1988.

[24] P. Frosst, H. J. Blom, R. Milos et al., "A candidate genetic risk factor for vascular disease: a common mutation in methylenetetrahydrofolate reductase," Nature Genetics, vol. 10, no. 1, pp. 111-113, 1995.

[25] S. Heux, F. Morin, R. A. Lea, M. Ovcaric, L. Tajouri, and L. R. Griffiths, "The methylentetrahydrofolate reductase gene variant $(\mathrm{C} 677 \mathrm{~T})$ as a risk factor for essential hypertension in Caucasians," Hypertension Research, vol. 27, no. 9, pp. 663667, 2004.

[26] I. Kara, A. Sazci, E. Ergul, G. Kaya, and G. Kilic, "Association of the C677T and A1298C polymorphisms in the 5,10 methylenetetrahydrofolate reductase gene in patients with migraine risk," Molecular Brain Research, vol. 111, no. 1-2, pp. 84-90, 2003.

[27] A. L. Szvetko, J. Fowdar, J. Nelson et al., "No association between MTHFR A1298C and MTRR A66G polymorphisms, and MS in an Australian cohort," Journal of the Neurological Sciences, vol. 252, no. 1, pp. 49-52, 2007.

[28] A. Feix, W. C. Winkelmayer, C. Eberle, G. Sunder-Plassmann, and M. Födinger, "Methionine synthase reductase MTRR $66 \mathrm{~A}>\mathrm{G}$ has no effect on total homocysteine, folate, and Vitamin B12 concentrations in renal transplant patients," Atherosclerosis, vol. 174, no. 1, pp. 43-48, 2004.

[29] L. Wang, Q. Ke, W. Chen et al., "Polymorphisms of MTHFD, plasma homocysteine levels, and risk of gastric cancer in a high-risk Chinese population," Clinical Cancer Research, vol. 13, no. 8, pp. 2526-2532, 2007.

[30] I. Menashe, P. S. Rosenberg, and B. E. Chen, "PGA: power calculator for case-control genetic association analyses," BMC Genetics, vol. 9, article 36, 2008.

[31] S. Purcell, B. Neale, K. Todd-Brown et al., "PLINK: a tool set for whole-genome association and population-based linkage analyses," The American Journal of Human Genetics, vol. 81, no. 3, pp. 559-575, 2007.

[32] J. K. Chiang, M. L. Sung, H. R. Yu et al., "Homocysteine induces smooth muscle cell proliferation through differential regulation of cyclins A and D1 expression," Journal of Cellular Physiology, vol. 226, no. 4, pp. 1017-1026, 2011.

[33] C. Munjal, S. Givvimani, N. Qipshidze, N. Tyagi, J. C. Falcone, and S. C. Tyagi, "Mesenteric vascular remodeling in hyperhomocysteinemia," Molecular and Cellular Biochemistry, vol. 348, no. 1-2, pp. 99-108, 2011.

[34] N. Ilhan, M. Kucuksu, D. Kaman, N. Ilhan, and Y. Ozbay, "The $677 \mathrm{C} / \mathrm{T}$ MTHFR polymorphism is associated with essential hypertension, coronary artery disease, and higher homocysteine levels," Archives of Medical Research, vol. 39, no. 1, pp. 125-130, 2008.

[35] X. Qian, Z. Lu, M. Tan, H. Liu, and D. Lu, "A metaanalysis of association between C677T polymorphism in the methylenetetrahydrofolate reductase gene and hypertension," European Journal of Human Genetics, vol. 15, no. 12, pp. 1239 1245, 2007.

[36] L. Chen, L. Liu, K. Hong, J. Hu, and X. Cheng, "Three genetic polymorphisms of homocysteine-metabolizing enzymes and risk of coronary heart disease: a meta-analysis based on 23 case-control studies," DNA and Cell Biology, vol. 31, no. 2, pp. 238-249, 2012.

[37] C. Scazzone, S. Acuto, E. Guglielmini, G. Campisi, and A. Bono, "Methionine Synthase Reductase (MTRR) A66G polymorphism is not related to plasma homocysteine concentration and the risk for vascular disease," Experimental and Molecular Pathology, vol. 86, no. 2, pp. 131-133, 2009.

[38] R. Kahleová, D. Palyzová, K. Zvára et al., "Essential hypertension in adolescents: association with insulin resistance and with metabolism of homocysteine and vitamins," American Journal of Hypertension, vol. 15, no. 10, pp. 857-864, 2002. 


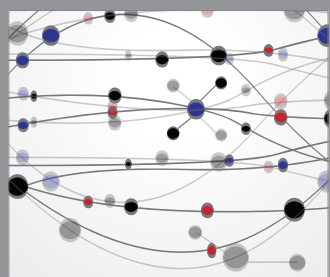

The Scientific World Journal
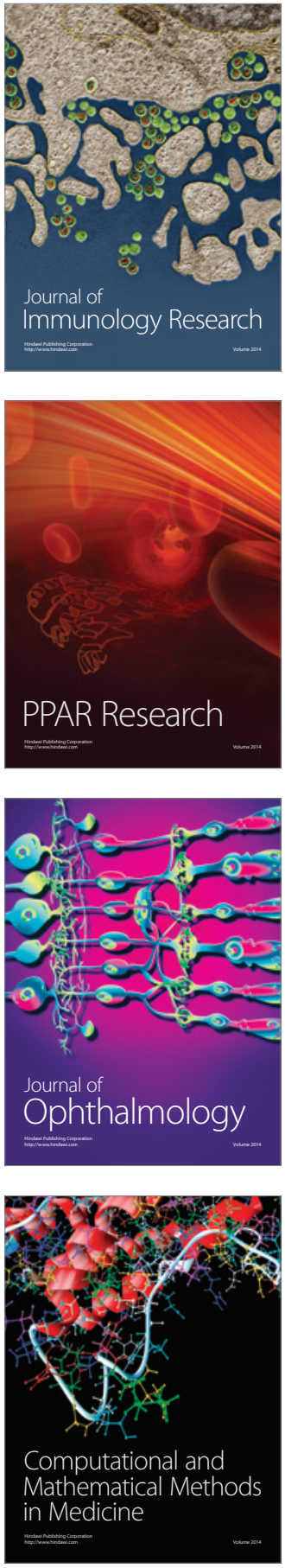

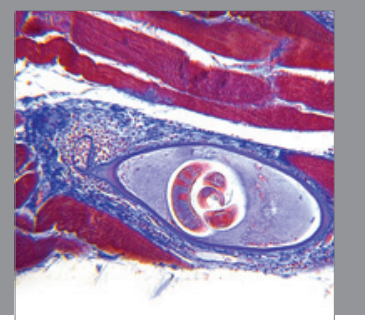

Gastroenterology

Research and Practice
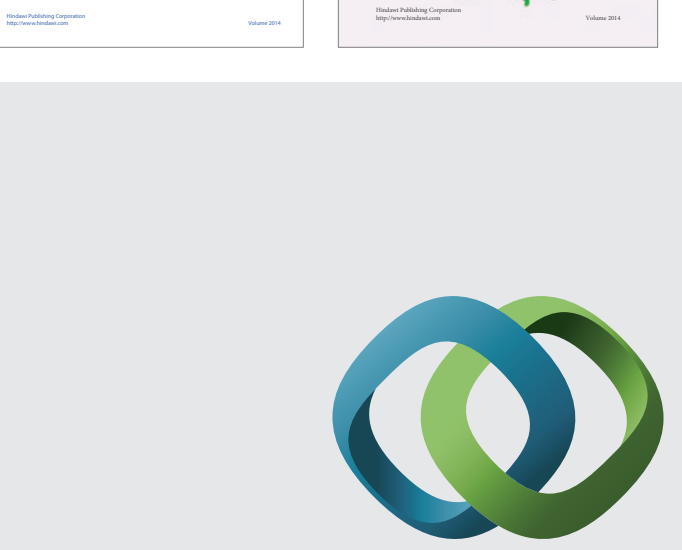

\section{Hindawi}

Submit your manuscripts at

http://www.hindawi.com
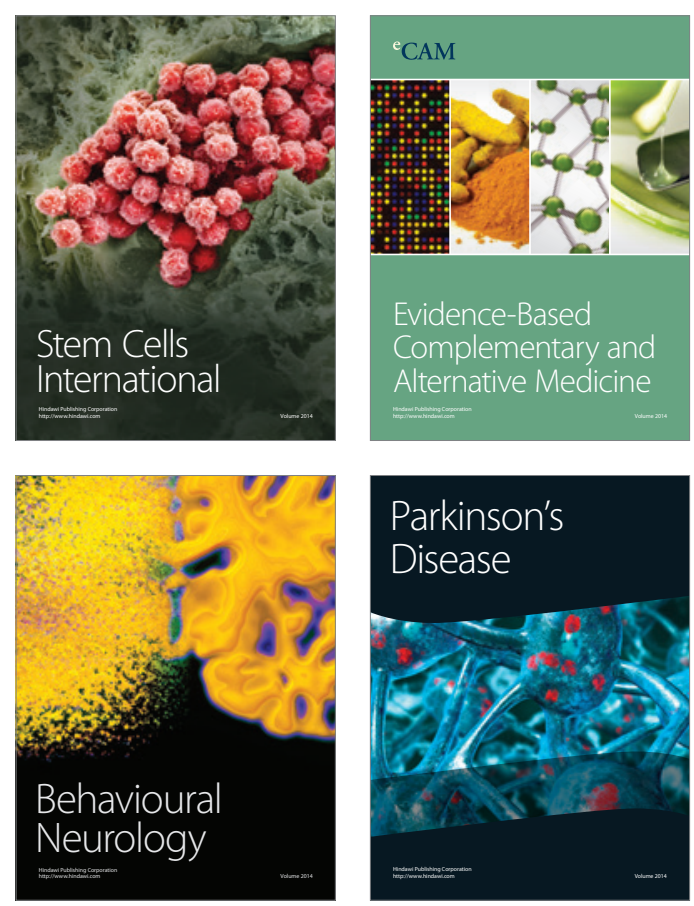

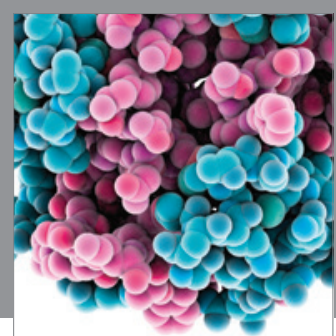

Journal of
Diabetes Research

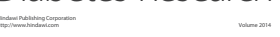

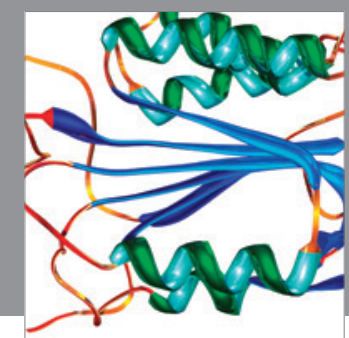

Disease Markers
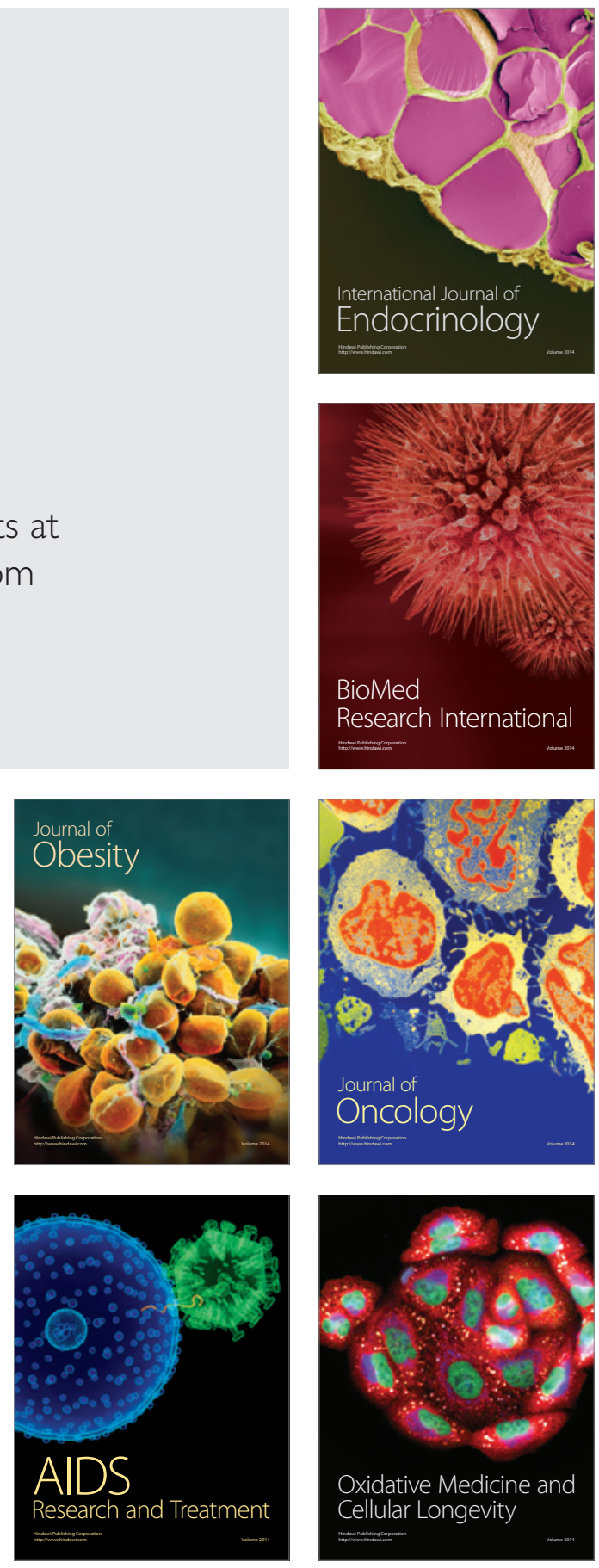